\title{
Thermal ellipsometry in steady-state and by lock-in thermography. Application to anisotropic materials characterization
}

\author{
by J.-C. Krapez, G. Gardette and D. Balageas
}

ONERA, OP/L3C, BP 72, F-92322 CHÂTILLON-Cedex, France.

\begin{abstract}
A technique for lock-in thermography that we recently developed was applied for the measurement of the in-plane principal diffusivities of orthotropic materials. The surface is point heated with a modulated laser beam and a 2-D synchronous detection is performed of the elliptical thermal field which develops around it. Diffusivities are inferred from the slope of the phase lag profiles. The approach was first validated with a duralumin (isotropic) sample. It was then applied on a unidirectional C/epoxy sample.

The aspect ratio of the surface isotherms is imposed by the anisotropy of the considered material. Therefore, possible in-depth variations of the anisotropy unavoidably modify the aspect ratio distribution at the surface. Based on this observation, an inversion method was recently proposed to monitor the internal fibre orientation changes in composites. Results obtained with a continuous fibres C/epoxy sample and with an injection moulded short fibres sample are reported.
\end{abstract}

\section{Introduction}

More than one century ago, de Sénarmont applied the following thermal approach to find out the principal orientations in crystal plates [1]: he covered them with a thin layer of wax, heated them over a small spot and monitored the isotherm shape revealed by the solid/liquid transition contour appearing in the wax layer. The isotherm proved to be elliptical and its aspect ratio was equal to the square root of the principal conductivities in the surface plane. This concept, to which we will refer as "Thermal Ellipsometry", was later used for various applications (with, of course, up-to-date experimental equipment): analysis of stretched polymers [2], evaluation of the average fibre orientation in carbon fibre composite samples [3], ... The steady-state or quasisteady-state regime was once considered. It however only led to the ratio of the principal conductivities in the sample plane (this information was recently obtained through line heating instead of point heating [4]). Other temporal regimes were then tried: the transient regime pertaining to step heating (with point heating $[5]$, with line or half-plane heating $[6,7])$ and the harmonic regime (with point heating [8-11], line heating [12], or half-plane heating [13]). They have the advantage to separately provide the values of the in-plane principal diffusivities: for this purpose, in the case of modulated heating, one may refer to the in-plane distribution of the phase lag or to its frequency dependence.

Point heating with a modulated laser beam and temperature monitoring by lock-in thermography probably represents the easiest and most rapid way to reveal material in-plane anisotropy and its possible in-depth variations $[14,15]$. We recently implemented a new approach for lock-in thermography [16]. Its advantages will be briefly outlined. We will present phase images which were obtained on isotropic and on anisotropic homogeneous materials. These images were further processed to infer in-plane diffusivity(ies) which were compared to the values obtained by the conventional flash method.

The transposition to the case of a laminate made of three orthotropic layers simulating the skin/core morphology of injection moulded composites will then be developed (indeed, as far as the fibre orientation is concerned, a stratification generally appears during the injection of this type of composite: near the mould surfaces the fibres get oriented in the direction of the flow, whereas in the middle they get oriented perpendicularly to this direction). We will finally present some results obtained with a newly devised method for the thickness evaluation of these layers. 


\section{Theory of thermal ellipsometry applied to homogeneous and orthotropic materials}

The temperature modulation over the isolated surface of a homogeneous and orthotropic halfspace with principal diffusivities $a_{x}, a_{y}, a_{z}$, which is heated with power $P$ and at frequency $f$ at point $(0,0)$ of its surface is expressed by [17]:

$$
T(x, y)=\frac{P}{2 \pi \rho C}\left[a_{x} a_{y} a_{z}\left(\frac{x^{2}}{a_{x}}+\frac{y^{2}}{a_{y}}\right)\right]^{-1 / 2} \exp \left[-(1+i) \sqrt{\pi f\left(\frac{x^{2}}{a_{x}}+\frac{y^{2}}{a_{y}}\right)}\right]
$$

Iso-level curves of the amplitude and of the phase maps are ellipses whose aspect ratio is equal to $A^{1 / 2}$ where $A$ is the anisotropy coefficient $a_{x} / a_{y}$ (it can be shown that this remains valid if one assumes that there are linear surface heat losses). The slope of the phase lag in the principal directions is:

$$
\frac{\partial \varphi}{\partial x_{i}}=-\sqrt{\frac{\pi f}{a_{i}}}
$$

However our samples fitted better to the model of a thin plate submitted to heat losses. The case of a point heated isotropic thin plate submitted to heat losses was already thoroughly discussed [9]. In this model, the heat transfer is considered to be one-dimensional (radial in-plane heat flow) The generalization to orthotropic plates is straightforward. In particular, the slope of the phase in the principal directions is found to be:

$$
\frac{\partial \varphi}{\partial x_{i}}=\operatorname{Im}\left[\beta \frac{K^{\prime} \alpha\left(\beta x_{i}\right)}{K_{0}\left(\beta x_{i}\right)}\right]
$$

where: $\quad \beta=\sqrt{\frac{i \omega}{a_{i}}+\frac{2 h}{e k_{i}}}$

$\mathrm{e}$ is the plate thickness and $\mathrm{h}$ is the coefficient of linear heat transfer. By using an asymptotic expansion of the Bessel functions, one finds out that for large values of $\beta x_{i}$ the phase slope can be approximated by:

$$
\frac{\partial \varphi}{\partial x_{i}} \approx-\operatorname{Im}(\beta) \cdot\left[1+\frac{1}{8\left|\beta^{2} x_{i}^{2}\right|}\right]
$$

However for moderate values of $\beta x_{i}$ Eq. 2 provides a better approximation than Eq. 5 . Assuming there are no heat losses, the actual phase slope is higher in absolute value, as compared to Eq. 2 , by $3 \%$ at $x_{i}=\mu_{i}$, and by $1 \%$ at $x_{i}=2 \mu_{i}$, where $\mu_{i}$ is the diffusion length in the considered direction, i.e. $\left(2 a_{i} / \omega\right)^{1 / 2}$. Inversely, the actual phase slope progressively decreases as heat losses become more important: at $x_{i}=\mu_{i}$, for example, the mismatch with the value given by Eq. 2 is $+0.5,-2$, and $-4.5 \%$ when $H$ is respectively equal to $0.05,0.1$, and 0.15 ( $\mathrm{H}$ corresponds to $2 h / \rho \mathrm{Ce} \omega)$.

In reality the sample is not point heated but it is heated over a finite area. If one assumes that the laser beam is Gaussian with a radius $r_{s}$ at $e^{-1 / 2}$ and that the IR detector used for the temperature monitoring integrates the incoming IR intensity over a Gaussian pattern with a radius $r_{d}$, the signal amplitude and phase will be determined by the "resolution radius" $r_{r}=\left(r_{s}{ }^{2}+r_{d}^{2}\right)^{1 / 2}$. There is a competition between the laser beam which would impose its circular shape to the isosignal curves (iso-amplitude as well as iso-phase) and the orthotropic material which would impose an elliptic shape to them. We showed that, in the limit of low frequencies, the aspect ratio of the iso-amplitude curves decreases from $A^{1 / 2}$, far from the heated spot, to about $A^{1 / 8}$ near its centre [18]. As far as the iso-phase curves are concerned it can be further shown that a $90^{\circ}$ rotation occurs near the centre for high frequencies: the central iso-phase curves are paradoxically oriented in the low diffusivity direction! This indicates that Eq. 2 cannot be used at the vicinity of the centre. We will therefore adopt the rule of thumb which was established for the case of isotropic materials, i.e. that the measurement of the phase slope should be done at a distance larger than $2 r_{r}$ from the heated spot [10]. 


\section{Experimental results with duralumin and C/epoxy samples}

The experimental set-up is depicted on figure 1 . Thin samples were heated with a $0.2-2 \mathrm{~W}$ Argon laser beam, about $0.8 \mathrm{~mm}$ in diameter which was square-modulated by a mechanical shutter. The samples were held horizontally in order to avoid convection-induced asymmetrical distortions of the temperature field. An infrared camera (Agema 880LW or Amber 4128) was used to record the temperature distribution evolution around the heated spot for a few periods.

Each thermographic film was subsequently processed to perform a 2-D synchronous detection (amplitude and phase images were retrieved therefrom). The developed algorithm is based on the maximum likelihood estimation criteria. The performances of this new method for lock-in themography were recently presented [16]. As compared to the approach promoted by Agema $[14,15,19]$, the choice for the modulation frequency is now greatly relaxed: the proposed method merely prohibits those modulation frequencies which are multiples of half the camera scanning frequency. Numerical simulations showed that through lock-in thermography, the signal to noise ratio $S / N$ is improved by a factor of between $(n / 2)^{1 / 2}$ and $(n / 4)^{1 / 2}$ where $n$ is the number of integrated images. As far as the phase is concerned, its standard deviation, expressed in degrees, is about $90 \cdot(\mathrm{S} / \mathrm{N})^{-1 /} / \mathrm{N}$.

We show in figure 2 the phase map obtained in the case of a $1 \mathrm{~mm}$ thick duralumin sample heated at $0.03 \mathrm{~Hz}$ frequency (a thin layer of black paint was added to increase the surface emissivity). Iso-level curves are found circular as the material is isotropic. Through a 2-D linear regression of the phase map (by considering only the pixels which were distant to the centre from 5.6 to $17 \mathrm{~mm}$ ), and by applying Eq. 2 with the global phase slope, we obtained, from a series of four tests performed at 0.03 and $0.02 \mathrm{~Hz}$, a diffusivity of $50.4 \pm 1.1 \cdot 10^{-6} \mathrm{~m}^{2} / \mathrm{s}$. This value is to be compared to $52.0 \pm 0.9 \cdot 10^{-6} \mathrm{~m}^{2} / \mathrm{s}$ which was obtained by a front-face laser flash method. The use of Eq. 2 was justified as heat losses had low impact (the coefficient $\mathrm{H}$ was estimated to be lower than 0.06 ). Furthermore, the phase slope was evaluated over a region which was remote from the heated spot (the distance was ranging from 0.2 to 0.7 times the thermal diffusion length; the identification however gave implicitly more weight to the slope that the phase lag reached far away). A diffusivity underestimation of not more than a couple of percent could thus occur. As far as the paint layer is concerned, its thickness, of the order of $20 \mu \mathrm{m}$, was too low to induce a significant phase lag.

Similar experiments were performed with a unidirectional C/epoxy sample, $1 \mathrm{~mm}$ thick, which was made of 8 plies of continuous carbon fibres. The phase map is reported on figure 2 for a modulation of $0.02 \mathrm{~Hz}$. The anisotropy of the material is clearly revealed. The inferred principal diffusivities are $3.85 \pm 0.12 \cdot 10^{-6} \mathrm{~m}^{2} / \mathrm{s}$ in the fibre direction and $0.67 \pm 0.04 \cdot 10^{-6} \mathrm{~m}^{2} / \mathrm{s}$ in the perpendicular direction. The inferred anisotropy coefficient, $A=5.7$, corresponds fairly well to the square of the aspect ratio of the isotherms which were obtained in the steady-state regime, i.e. $6.4 \pm 0.1$. We also measured the diffusivity in the direction normal to the surface by applying the conventional rear-face flash method and obtained $0.44 \pm 0.02 \cdot 10^{-6} \mathrm{~m}^{2} / \mathrm{s}$. This diffusivity is $35 \%$ lower than the one obtained in the plane and across the fibres: this is due to the fabrication process (autoclave) which left resin-rich sub-layers between the plies, and introduced thereby thermal resistances parallel to the surface.

\section{Theory of thermal ellipsometry applied to orthotropic laminates}

The purpose was then to use themal ellipsometry to characterize materials having the 3-layer morphology as mentioned before. The materials of interest were expected to have uniform thermal properties in the direction normal to the surface. Classical methods relying on uniform surface heating could not thus be used to identify the skin and core layers. On the contrary, thermal ellipsometry was expected to be of great help: indeed, near the heated point the isotherms should be mainly influenced by the upper skin layer anisotropy, and the core layer should influence only wider isotherms [20]. The analysis of the isotherm aspect ratio distribution around the heated spot should provide insight on the depth of the two skin/core interfaces. First theoretical results obtained with a 3-D analytical model of heat transfer in orthotropic laminates showed the potential of the method $[18,21]$. We recall on figure 3 the results obtained for the particular case of symmetrical stackings with a core relative thickness of $1 / 3$ and $2 / 3$. Steady- 
state regime was simulated with a Biot number he/ $k_{z}$ of 0.05 , a resolution radius of $0.2 e$, and a nominal anisotropy coefficient for each layer of $A=6.4$ (typical for C/epoxy). The aspect ratio of the isotherms $r_{x} / r_{y}$ was reported vs. their relative mean radius, i.e. $\left(r_{x} r_{y}\right)^{1 / 2} / e$. The aspect ratio of the smallest isotherms is dictated by the heating spot shape and size (if it were punctual, this aspect ratio would be of $A^{1 / 2}=2.5$ ). Then a transition region reveals the presence of the core layer at $90^{\circ}$ to the skin layer. Its radius extends from about 0.3 to 10 times the total thickness. Then, the aspect ratio reaches an asymptotic value $F$ which is merely representative of the core relative thickness $e_{c} / e$ :

$$
\frac{e_{c}}{e}=\left(A-F^{2}\right)(A-1)^{-1}\left(F^{2}+1\right)^{-1}
$$

We conciude that only the isotherms of the transition region can help to identify the core depth.

In the case of modulated heating, the influence of deep layers on the surface aspect ratio of the iso-amplitude curves and of the iso-phase curves having a given mean radius progressively diminishes as frequency increases (see figure 4). Actually, by modulating the heat input, one merely spreads the information on internal anisotropy changes over a wider surface field. The only advantage would be that, by relying on the phase map instead of the temperature map, one would get rid of the spurious influence of the emissivity variations which possibly affect the surface. This is a well known advantage for the use of phase maps [15].

An inversion method was devised to infer both the core layer depth and its thickness from the steady-state thermal field by fitting experimental and theoretical distributions of the isotherm aspect ratio [22]. Figure A (colour plate) indicates the IR images obtained with four symmetrical $\mathrm{C} /$ Epoxy samples $2.2 \mathrm{~mm}$ thick which were made of continuous fibres plies and where the core relative thickness was $1 / 6,1 / 3,1 / 2$, and $2 / 3$.

After inversion, the retrieved values of the relative thickness and depth were as reported in Table 1 . The results about core thickness are reasonably precise (less than about $5 \%$ error). However, the precision of its mean depth gets less satisfactory as the upper interface gets deeper into the composite.

Thermal ellipsometry was also performed at several locations of an injection moulded C/PEEK sample containing short fibres. The isotherms were all elongated in the flow direction, thus indicating that the core layer was everywhere less than 0.5 thick. After inversion the core layer was generally found nearer the upper surface than it was in reality (as revealed by microscopy after sectioning the sample). Anyway, the calculated core thickness variations across the sample were in close relation with their actual counterpart. Furthermore, a significant correlation was found between the mean aspect ratio at each test location and the flexural modulus measured on coupons extracted therefrom (correlation coefficient of 0.96 ), thus highlighting the potential of thermal ellipsometry for the quality assessment of injection moulded composites made of carbon fibres.

\section{Acknowledgements}

This work was supported by the Commission of the European Communities DGXII under the Brite/Euram contract nBR-2-CT02-0139.

\section{REFERENCES}

[1] DE SÉNARMONT (M.H.). Annales de Chimie Physique, vol. 3(2), 1848, p. 179-211.

[2] PIETRALLA (M.). Colloid Polym. Sci., vol. 259, 1981, p. 111-129.

[3] FOLKES (M.J.) and POTTS (H.A.). Plastics and Rubber Proc. and Appl., vol. 10, 1988, p. 79-84.

[4] RANTALA (J.). Rev. Sci. Instrum., vol. 63(11), 1992, p. 5472-5474.

[5] BLUM (K.), KILIAN (H.G.) and PIETRALLA (M.). J. Phys. E, Sci. Instrum., vol. 16, 1983, p. 807-812. 
[6] WELCH (C.S.), HEATH (D.M.) and WINFREE (W.P.). J. Appl. Phys., vol. 61(3), 1987, p. 895-898.

[7] PHILIPPI (I.), BATSATE (J.C.), MAILLET (D.) and DEGIOVANNI (A.). Rev. Sci. Instrum., vol. 66(1), 1995, p. 182-192.

[8] BUSSE (G.), RIEF (B.) and EYERER (P.). Polymer Composites, vol. 8(4), 1987, p. 283286.

[9] VISSER (E.P.), VERSTEEGEN (E.H.) and VAN ENCKEVORT (W.J.P.). J. Appl. Phys., vol. 71(7), 1992, p. 3238-3248.

[10] LEPOUTRE (F.), BALAGEAS (D.), FORGE (P.), HIRSCHI (S.), JOULAUD (J.L.), ROCHAIS (D.) and CHEN (F.C.). J. Appl. Phys., vol. 78(4), 1995, p. 2208-2223.

[11] GRÖNBECK (H.) and REICHLING (M.). J. Appl. Phys., vol. 78(11), 1995, p. 6408-6413.

[12] KOSKY (P.G.). Rev. Sci. Instrum., vol. 64(4), 1993, p. 1071-1075.

[13] CHEN (G.), TIEN (C.L.), WU (X.) and SMITH (J.S.). J. Heat Transfer, vol. 116, 1994, p. 325-331.

[14] KARPEN (W.), WU (D.), STEEGMÜLLER (R.) and BUSSE (G.). Proc. QIRT 94 Conf., eds. D. Balageas, G. Busse, and G.M. Carlomagno (Editions Européennes Thermique et Industrie, Paris, 1995), 1995, p. 281-286.

[15] WU (D.), STEEGMÜLLER (R.), KARPEN (W.) and BUSSE (G.). Review of Progress in QNDE, vol. 14, eds. D.O. Thompson and D.E. Chimenti (Plenum, New York), 1995, p. 439-446. [16] KRAPEZ (J.-C.), GARDETTE (G.) and BALAGEAS (D.). Proc. 3rd Int. Workshop on Advanced IR Techn. and Appl., Capri (It.), Sept. 19-20 1995, ed. Fondazione G. Ronchi (Firenze), 1995, p. 219-237.

[17] CARSLAW (H.S.) and JAEGER (J.C.). Conduction of heat in solids, 2nd. ed. (Oxford University Press), 1959.

[18] KRAPEZ (J.-C.). Report RT91-010-121-02, IMI, National Research Council Canada, 1991.

[19] BUSSE (G.), WU (D.) and KARPEN (W.). J. Appl. Phys., vol. 71(8), 1992, p. 3962-3965.

[20] KRAPEZ (J.-C.), CIELO (P.), MALDAGUE (X.) and UTRACKI (L.A.). Polym. Comp., vol. 8(6), 1987, p. 396-407.

[21] KRAPEZ (J.-C.). Journée d'Etude SFT sur la Thermographie Quantitative, 7 déc. 1994.

[22] KRAPEZ (J.-C.). Review of Progress in QNDE, vol. 15, eds. D.O. Thompson and D.E. Chimenti (Plenum, New York), 1996, p. 533-540.

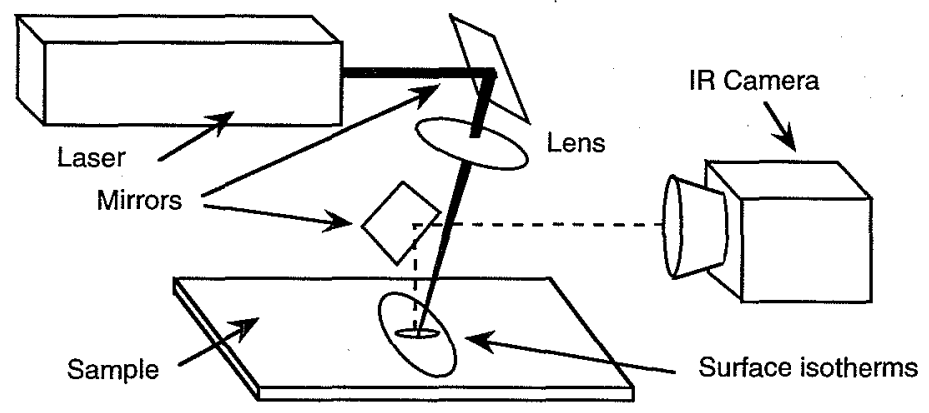

Fig. 1. Experimental set-up for thermal ellipsometry. 

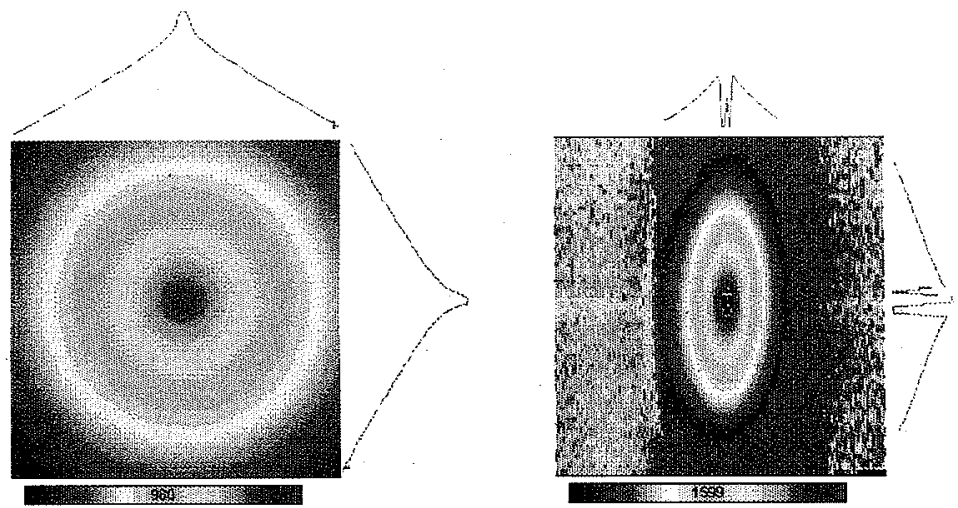

Fig. 2. Phase lag maps obtained by modulated thermal ellipsometry on a duralumin sample (left) and on a unidirectional Clepoxy sample (right). IR camera field was $44 \mathbf{~ m m}$ wide.

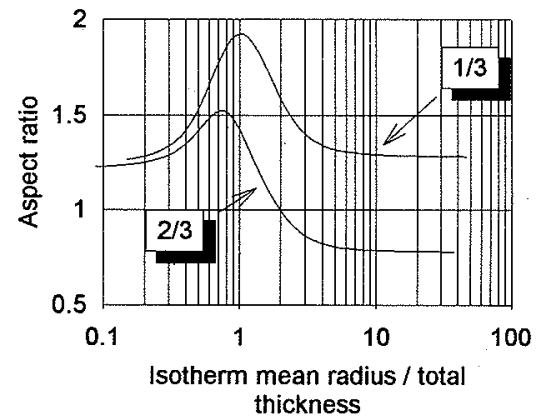

Fig. 3. Isotherm aspect ratio profiles obtained in steady-state regime in the case of two symmetrical skin/core/skin stackings (core oriented at $90^{\circ}$ to the skin). Core relative thickness of either $1 / 3$ or $2 / 3$. The "resolution radius" corresponds to 0.2 times the laminate thickness.

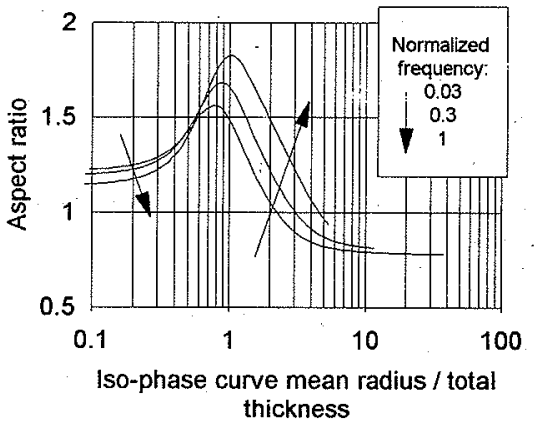

Fig.4. Same as fig. 3 for the iso-phase curves obtained with a $2 / 3$ thick core layer in the case of modulated heating. Different values of the reduced frequency $\mathrm{fe}^{2} / \mathrm{a}_{\mathrm{z}}$ are considered.

Table 1. Results of the inversion of the IR images presented on fig. A (colour plate)

\begin{tabular}{ccc}
\hline Core layer actual thickness & Calculated thickness & Calculated mean depth \\
\hline & & \\
0.167 & $0.146 \pm 0.013$ & $0.664 \pm 0.050$ \\
0.333 & $0.341 \pm 0.021$ & $0.501 \pm 0.024$ \\
0.500 & $0.553 \pm 0.015$ & $0.581 \pm 0.016$ \\
0.667 & $0.694 \pm 0.019$ & $0.533 \pm 0.015$ \\
\hline
\end{tabular}

